\section{Schimmelpenning-Feuerstein- Mims-Syndrom in Kombination mit einem primären Lymphödem und Basalzellkarzinomen}

Zusammenfassung. Es wird über einen 69-jährigen Patienten mit einem ausgedehnten Schimmelpenning-Feuerstein-MimsSyndrom und primärem Lymphödem der linken unteren Extremität sowie Genital, Mundschleimhautbeteiligung und Iriskolobom berichtet. Als Spätkomplikation entwickelten sich auf dem organoiden Naevus benigne und ausgedehnte maligne Sekundärproliferationen.

Schimmelpennig-Feuerstein-Mims-Syndrome with Lymphedema and Basal Cell Carcinomas. A 69year old man with an unilateral extensive Schimmelpennig-Feuerstein-Mims-Syndrome presented with lymphedema of the left lower limb and genital region, mucous membrane affection and iris colobom. He also developed both benign and malignant secondary tumors within the nevus as late complications.

\section{Einleitung}

Das Schimmelpenning-Feuerstein-Mims-Syndrom (SFMS) gehört zur Gruppe der Phakomatosen und wurde erstmals von Schimmelpenning [1] 1957 sowie Feuerstein und Mims [2] 1961 beschrieben. Leitsymptom des SFMS ist ein striärer, halbseitig lokalisierter, teilweise auch multilokulärer Naevus sebaceus, der entsprechend der Blaschkoschen Linien verteilt ist. Der Naevus sebaceus ist bereits beim Neugeborenen manifest, wobei seine organoide Differenzierung aber einer weiteren Entwicklung unterliegt, die besonders durch hormonelle Einflüsse in der Pubertät gesteuert wird [3-5].

Ein genetischer Basisdefekt ist beim SFMS bislang nicht bekannt. Aufgrund des sporadischen Auftretens des Syndroms, des Verteilungsmusters und der unterschiedlichen Ausprägung der einzelnen Symptome wird die Wirkung eines letalen autosomalen Gens postuliert, welches durch eine Mosaik-Verteilung manifest wird $[4,6]$.

Akt Dermatol 2001; 27: 66-69

(c) Georg Thieme Verlag Stuttgart · New York ISSN 0340-2541
W. Harth, R. Linse

Klinik für Hautkrankheiten, Klinikum Erfurt

(Direktorin: Prof. Dr. Ruthild Linse)
Im vorliegenden Fall wird die sehr ausgedehnte Form eines SFMS mit ausgedehntem Lymphödem und dem Auftreten von Basalzellkarzinomen beschrieben.

\section{Kasuistik \\ Familienanamnese}

Bei den Eltern des 69-jährigen männlichen Patienten und der gesunden Tochter besteht kein Schimmelpenning-FeuersteinMims-Syndrom. Die körperliche Untersuchung der Tochter zeigt keine abortiven Symptome des SFMS.

\section{Anamnese}

Bei der Geburt besteht ein segmental angeordnetes, auf die linke Körperhälfte begrenztes anfänglich gelb-rötliches Hautinfiltrat, und seit der Kindheit eine langsam progrediente Schwellung des linken Beines mit zunehmender Ausdehnung auf den Genitalbereich. Seit 1990 entwickeln sich auf den jetzt verrukösen Hautveränderungen im Gesichtsbereich Knoten, die bei fehlendem Leidensdruck monströse Ausmaße annehmen. Nach Exzision erfolgt eine Defektdeckung mittels Nahlappenplastik sowie paramedian gestieltem Stirnlappen und anschließender Rückverlagerung.

\section{Dermatologischer Untersuchungsbefund}

Auf der linken Körperhälfte erstrecken sich vom Capillitium bis zum Fuß, entsprechend der Blaschkoschen Linien angeordnete, bizarr konfigurierte Hautläsionen von gelblich-hellbraunem Kolorit und feingefurchter bis papillomatöser, hyperkeratotischer Oberflächenstruktur (Abb. 1 u. 2). In diese Areale eingestreut sind zahlreiche dunkelbraune bis schwärzliche, im Durchmesser $1-2 \mathrm{~cm}$ große, verruköse Knoten. Die linke untere Extremität einschließlich des Fußes weist insgesamt eine lymphomatöse derbe Schwellung auf. Im Oberschenkelbereich besteht zur Gegenseite eine Umfangsdifferenz von $19 \mathrm{~cm}$. Links besteht eine klumpfußartige Fußdeformierung. Penis und Skrotum zeigen ein massives Lymphödem und sind entzündlich gerötet. Im distalen Skrotumbereich sind einige lymphokutane, sezernierende Fisteln erkennbar.

Im Gesichtsbereich erstreckt sich der Naevus auf die gesamte linke Gesichtshälfte mit Übergang auf das Capillitium, wo okzipital ein $5 \times 8 \mathrm{~cm}$ großer Alopeziebezirk ausgeprägt ist. An 

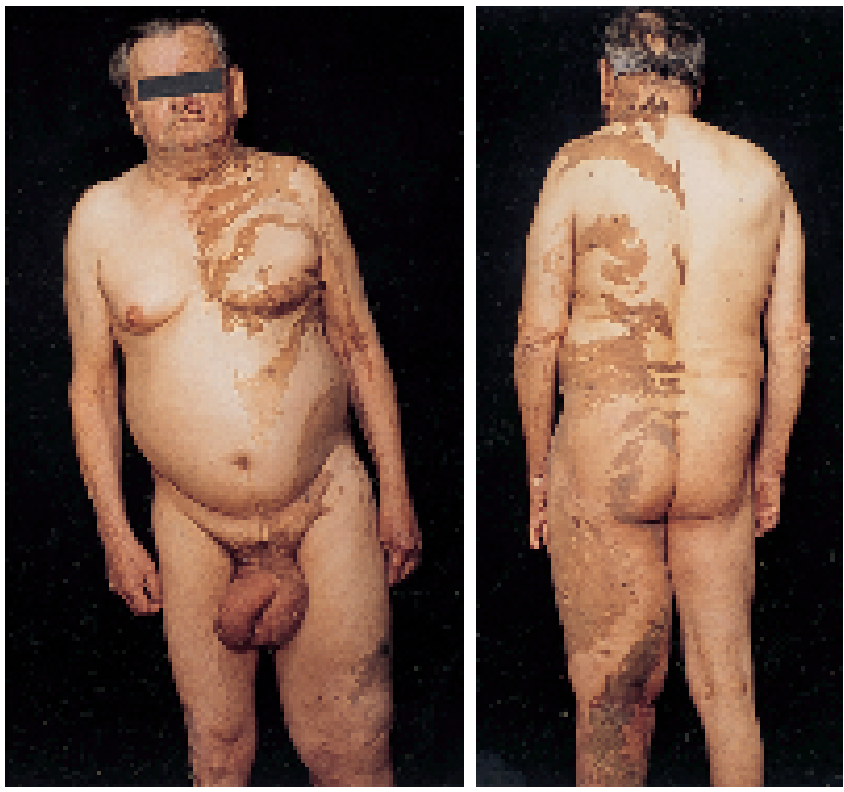

Abb. 1 SFMS: Übersicht, frontal.

Abb. 2 SFMS: Übersicht, dorsal.

der linken Wangenschleimhaut findet sich eine striär, weißlich, verruköse Proliferation. Es besteht eine Hemimakroglossie und Lingua plicata links. Vom Nasenflügel über die Nasolabialfalte bis zum linken Mundwinkel sind vier rötliche, prall elastische, halbkugelige Koten (Abb. 3) aneinandergereiht. Die perlmuttartig glänzende Oberfläche zeigt multiple Teleangiektasien und zentrale, verkrustete Ulzerationen. Die Tumoren am Nasenflügel sind zweikugelig auf einer gemeinsamen Basis und $1,5 \mathrm{~cm}$ und $3,5 \mathrm{~cm}$ im Durchmesser groß sowie die angrenzenden Tumoren $0,6 \mathrm{~cm}$ und $3 \mathrm{~cm}$.

\section{Befunde}

Laborbefunde: Routine-Laborparameter im Normbereich.

Sonographie Abdomen: Hepatomegalie mit Steatosis hepatis.

Röntgen-Thorax und untere Extremitäten: Rechts konvexe Skoliose der Brustwirbelsäule. Coxarthrose und Gonarthrose beidseits. Linke Tibia kräftiger strukturiert als rechts.

Computertomographie Schädel: Seitengleiche Zeichen der kortikalen Atrophie. Es zeigt sich eine deutliche Verdickung der Schädelkalotte frontoparietal rechts.

Elektroenzephalographie: Bitemporal betonte unspezifische Störungen und parieto-okzipital rechts betonte Alpha-Wellen Reduktion.

Ophthalmologischer Befund: Peripheres Iriskolobom am linken Auge sowie Aphakie bei Zustand nach Kataraktoperation.

\section{Histopathologische Befunde}

1. Probebiopsie Capillitium: Naevus verrucosus et sebaceus

2. Nuchal und

3. linke Oberarminnenseite: Verrucae seborrhoicae

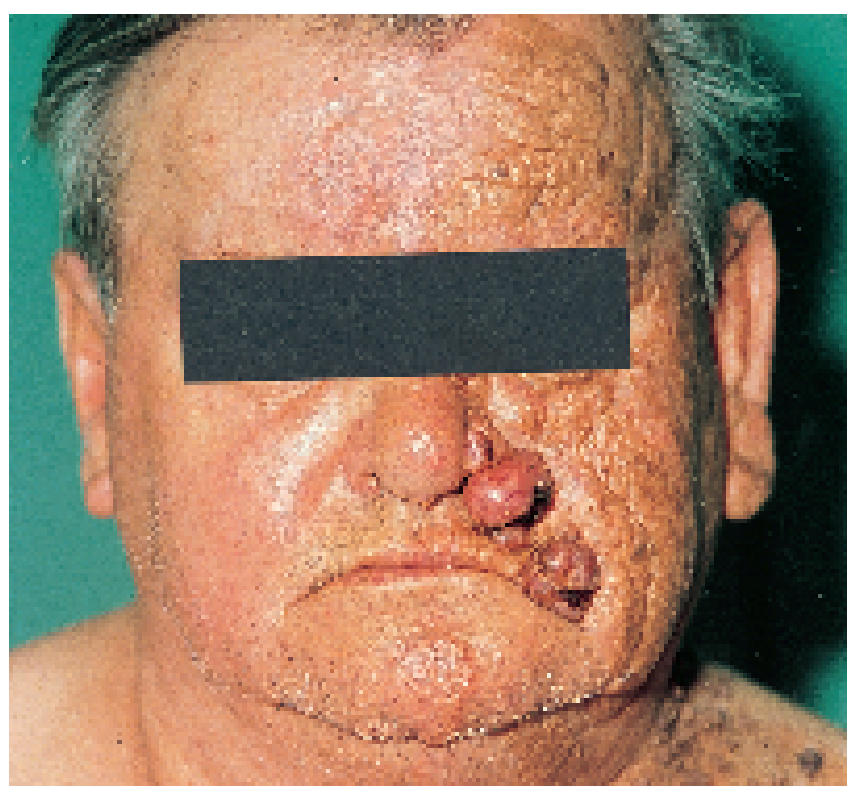

Abb. 3 SFMS: Gesicht: Basalzellkarzinome unterschiedlicher Differenzierung.

4. Nasenflügel und Nasolabialfalte links: zweigeteiltes verhorntes Basalzellkarzinom

5. Oberlippe links: oberflächlich ulzeriertes Basalzellkarzinom

6. Mundwinkel links: Adenoid zystisches, noduläres Basalzellkarzinom

7. Nuchal, lateral links: Fibroepitheliom

\section{Diskussion}

Aus der Gruppe der epidermalen Naevussyndrome stellt das SFMS ein gut charakterisiertes neuro-ektodermales, zum Teil auch mesodermales Missbildungssyndrom dar $[2,7,8]$. Zu dem Syndrom gehören charakteristische Fehlbildungen [3, 6, 9-11] von Haut, Augen, Hirn und Herz (HAHH-Syndrom). Es werden auch Missbildungen mesodermaler Strukturen insbesondere des Knochensystems beschrieben (Tab. 1). Differenzialdiagnostisch lassen sich weitere Epidermale Naevus Syndrome vom SFMS abgrenzen. Das CHILD-Syndrom (congenital hemidysplasia with ichthyosiform nevus and limb defects) ist durch einen großflächigen, entzündlich ichthyosiformen Naevus und Hypoplasie von Extremitäten charakterisiert sowie das Proteus-Syndrom mit flachem epidermalen Naevus und Makrodaktylie. Weiterhin wird diskutiert, das Naevus-comedonicus-Syndrom und das Becker-Naevus-Syndrom zur Gruppe der Epidermalen Naevus-Syndrome [4] zu zählen.

Aufgrund des genetischen Mechanismus wird die Phacomatosis pigmentokeratotica [12] abgegrenzt, wobei zusätzlich zu einem organoiden Naevus mit Talgdrüsendifferenzierung ein lentiginöser Naevus spilus besteht.

Bei dem von uns vorgestellten Patienten findet sich ein ausgedehnter, streng halbseitig lokalisierter, multilokulär und entsprechend der Blaschkoschen Linien angeordneter Naevus sebaceus. Zusätzlich bestehen eine Hemimakroglossie und Lingua plicata links und an der linken Wangenschleimhaut eine striär, weißlich, verruköse Proliferation. Ein solcher ausgedehnter Befall der Mundschleimhaut wurde bereits von Horn- 
Tab. 1 Schimmelpennig-Feuerstein-Mims-Syndrom: Assoziierte Fehlbildungen

\begin{tabular}{lll}
\hline Zentralnervensystem & Auge & mesodermales Keimblatt \\
\hline Hemiparesen & Mikrophthalmus & Hyperostosen/Asymmetrien \\
Epilepsie & Iris-Kolobom & Missbildungen im Herz-Kreislaufsystem \\
geistige Retardierung & Nystagmus & Missbildungen der Nieren \\
EEG-Veränderungen & Hornhauttrübung & Lymphödeme \\
\hline
\end{tabular}

stein beobachtet [13]. Aus ophthalmologischer Sicht liegt eine einseitige Aphakie links bei Zustand nach Katarakt-OP und ein beim SFMS häufig zu beobachtendes Iriskolobom vor. Mikrophthalmus, Nystagmus oder Hornhauttrübungen [13,14] fanden sich nicht. Im EEG konnten bei dem Patienten Störungen, betont rechts temporal, nachgewiesen werden. Als Zeichen einer Mitbeteiligung des Zentralnervensystems können EEGVeränderungen, Hemiparesen, epileptische Anfälle und eine geistige Retardierung auftreten $[2,15,16]$, die ihre volle Ausprägung erst im Laufe der Zeit entwickeln. Es zeigte sich weiterhin eine kontrolaterale Verdickung der Schädelkalotte frontoparietal rechts und eine kortikale Atrophie. Im Bereich der knöchernen Strukturen wurde über Schädelasymmetrien und Hyperostosen berichtet, die in der Regel ipsilateral vorkommen $[1,13,17]$, aber auch ipsi- und kontrolateral auftreten $[3,14]$.

Als mesodermale Missbildung imponiert weiterhin ein massives Lymphödem der gesamten linken unteren Extremität und des Genitalbereiches einschließlich entlastender Fisteln im Skrotalbereich. Die Assoziation eines SFMS mit einem ipsilateralen Lymphödem ist unseres Wissens nach bisher noch nicht beschrieben worden. Die Beteiligung des Lymphgefäßsystems in Form von Hypo- und Hyperplasie der Lymphgefäße [18] könnte ein neues mesodermales assoziiertes Symptom des SIMS darstellen.

Unser 69-jähriger Patient mit SFMS entwickelte, als dermatologische Spätkomplikation, im Bereich des Naevus verrucosus et sebaceus mehrere unterschiedliche Sekundärproliferationen.

Auf dem Boden des generalisierten Naevus verrucosus et sebaceus bestanden simultan Basalzellkarzinome verschiedener Differenzierungsformen, Fibroepitheliome und Verrucae seborrhoicae.

Während der Naevus sebaceus bei der Mehrzahl der Patienten im späteren Leben nahezu unverändert bestehen bleibt, zeigten sich im Rahmen einer Auswertung des Krankengutes an der Hautklinik Erfurt bei 57 (28,5\%) von 200 Patienten (1960-1980) Sekundärproliferationen [19,20]. Hinsichtlich der Art der Sekundärproliferation sind unterschiedliche Neoplasien entsprechend der verschiedenen naevoiden Gewebsanteile zu verzeichnen. Das Basaliom (26,3\%) einschließlich der basaloiden Differenzierungen $(28,1 \%)$ und das Syringozystadenoma papilliferum $(21,1 \%)$ waren am häufigsten unter allen Sekundärproliferationen $(n=57)$ vertreten. Weiterhin fanden sich seltene Proliferationen: Talgdrüsenepitheliome $(5,2 \%)$, Hidradenome $(3,5 \%)$ und intraepitheliales Epitheliom Borst-Jadassohn (1,8\%). Die Entwicklung eines Kar- zinoms auf dem Boden eines Naevus sebaceus wurde einmalig (1,8\%) beobachtet.

Demnach ist der Naevus sebaceus und insbesondere das großflächige Schimmelpenning-Feuerstein-Mims-Syndrom als fakultative Präkanzerose einzuordnen. Schon im ersten Lebensjahrzehnt und besonders mit dem Eintritt in die Adoleszenz können sich - ausgehend von der Epidermis und den Hautanhangsgebilden-benigne und maligne Neoplasien entwickeln. Prophylaktisch sollten aus diesem Grund kleinere Naevi sebacei bereits frühzeitig operativ entfernt werden. Da beim SFMS die vollständige Exzision aufgrund der großflächigen Ausdehnung meistens nicht möglich ist, sollte eine lebenslange Verlaufskontrolle beim Dermatologen erfolgen, um gegebenenfalls Sekundärproliferationen rechtzeitig operativ entfernen zu können. Die Prognose und der Verlauf des SFMS ist von der Ausprägung der Hautveränderungen, assoziierten Missbildungen sowie den daraus hervorgehenden Spätkomplikationen abhängig, und wird ebenfalls vom Zeitpunkt der Diagnosestellung sowie der daraus resultierenden kontinuierlichen interdisziplinären Langzeitbetreuung bestimmt. Nur so können Spätkomplikationen gering gehalten, maligne Sekundärproliferationen rechtzeitig erkannt und ausgedehnte Eingriffe vermieden werden.

\section{Literatur}

${ }^{1}$ Schimmelpennig GW. Klinischer Beitrag zur Symptomatologie der Phakomatosen. Fortschr Röntgenstr 1957; 87: 716-720

2 Feuerstein RC, Mims LC. Linear nervus sebaceus with convulsions and mental retardation. Amer J Dis Child 1962; 104: 675-679

${ }^{3}$ Fahnenstich H, Biltz H, Kreysel HW. Organoides Naevussyndrom (Schimmelpennig-Feuerstein-Mims-Syndrom): Kasuistik und Literatur. Klin Paediatr 1989; 201: 54-57

${ }^{4}$ Happle R. Epidermal nevus syndromes. Semin Dermatol 1995; 14: $111-121$

${ }^{5}$ Steigleder GK, Cortes AC. Verhalten der Talgdrüsen im Talgdrüsennävus während des Kindesalters. Arch Klin Exp Dermatol 1971; 239: $323-328$

${ }^{6}$ Happle R. Lethal genes surviving by mosaicism: a possible explanation for sporadic birth defects involving the skin. J Am Acad Dermatol 1987; 14: 899-906

${ }^{7}$ Lentz CL, Altmann J, Mopper C. Nevus sebaceus of Jadassohn. Report of one case with multiple and extensive lesions and an unusual distribution. Arch Dermatol 1968; 97: 294-296

8 Pinkus H. Organoid nevus. Mod Probl Paediatr 1976; 20: 50-57

${ }^{9}$ Barth PG, Valk J, Kalsbeek GL, Blom A. Organoid nevus syndrome: clinical and radiological study of a case. Neuropediatr 1977; 8: $418-428$

${ }^{10}$ Leyh F, Loewel R. Ein Fall von Schimmelpennig-Feuerstein-MimsSyndrom mit allmählicher Kataraktentwicklung. Z Hautkr 1973; 48: $695-698$

11 Solomon LW, Fretzin DF, Dewald RL. The epidermal nevus syndrome. Arch Derm 1968; 97: 273 - 285 
${ }^{12}$ Tadini G, Restano L, Gonzales-Peres R, Gonzales-Esenat A, Vincente-Villa A, Cambiaghi S, Marchettini P, Mastrangelo M, Happle R. Phacomatosis Pigmentokeratotica. Arch Dermatol 1998; 134: $333-337$

${ }^{13}$ Hornstein OP, Knickenberg M. Zur Kenntnis des Schimmelpennig-Feuerstein-Mims-Syndroms. Arch Derm Forsch 1974; 250: $33-50$

${ }^{14}$ Moynahan EJ, Wolff OH. A new neuro-cutaneous syndrome (skin, eye and brain) consisting of linear naevus, bilateral lipo-dermoid of the conjunctivae, cranial thickening, cerebral cortical atrophy and mental retardation. Brit J Derm 1967; 79: 651 -652

${ }^{15}$ Geissler H. Systematisierter Naevus sebaceus in Kombination mit epileptiformen Krampfanfällen. Derm Wschr 1966; 152: 1291

${ }^{16}$ Schimmelpennig GW. Langjährige Verlaufsuntersuchung einer organoiden Nävusphakomatose (Schimmelpennig-FeuersteinMims-Syndrom). Fortschr Röntgenstr 1983; 139: 63-67

${ }^{17}$ Ehlers E. Sekundärproliferationen auf Naevus sebaceus. Diplomarbeit. Erfurt: Medizinische Akademie, 1983

${ }^{18}$ Földi M, Casley-Smith JR. Lymphangiology. Stuttgart: Schattauer, 1983

${ }^{19}$ Cribier B, Scrivener Y, Grosshans E. Tumors arising in nevus sebaceus: A study of 596 cases. J Am Acad Dermatol 2000; 42: 263 268

${ }^{20}$ Linse R, Hadlich J, Ehlers E, Schubert H. Sekundärproliferationen auf Naevus sebaceus. Dermatologische Monatsschrift 1989; 173: $153-157$
Dr. med. Wolfgang Harth

Hautklinik

Klinikum Erfurt

Arnstädter Straße 34

99096 Erfurt

E-mail: Wolfgang.Harth@t-online.de 